\title{
Branch Unit for Seafloor Observatory Network
}

\author{
Yingbin Feng ${ }^{1,2}$, Zhigang Li $^{1}$, Xiaohui Wang ${ }^{1}$ \\ ${ }^{1}$ (Shenyang Institute of Automation, Chinese Academy of Sciences, Shenyang, China) \\ ${ }^{2}$ (Graduate School of the Chinese Academy of Sciences, Beijing, China) \\ Email: fengyb@sia.cn
}

\begin{abstract}
One key issue of seafloor observatory network is to ensure that the whole system operates normally when some equipment failure exists. Branch Unit can cut off the connection between the failure parts and the backbone network automatically and protect other equipment of the network from the impact of equipment failure. This paper proposes a design of the Branch Unit. The Branch Unit uses zener diode to provide power for the control circuits and constructs DWDM system with the help of the OADM. One Branch Unit model was built in this paper. Experiments verify the principle and functions of the Branch Unit and prove the feasibility of the design. The design provides a reference for the realization of the seafloor observatory network.
\end{abstract}

Keywords-seafloor obseratory network; Branch Unit; DWDM; zener diode

\section{INTROUCTION}

Oceans cover 70 percent of the earth's surface; the seafloor is rich in natural resources such as manganese nodules, hydrothermal sulfide, oil and gas, and natural gas hydrate. Exploration and exploitation of marine resources is very important for human survival and sustainable development [1]. Following the ground/ocean and space, seafloor observatory network becomes the third platform of observing the earth system. The North Eastern Pacific TimeSeries Undersea Networked Experiment (NEPTUNE) and ESONET are two sub-sea networks, which were initiated by the USA and Canada (NEPTUNE) and European Union respectively [2-3]. Seafloor observatory network is gradually becoming a new hot spot in the field of marine science and technology. Compared with foreign countries, seafloor observatory network construction starts relatively late in China. But some important progress in some key technology has yet been made.

Seafloor observatory network has broken the constraints of energy and data transmission, and makes that it is possible to obtain long-term, real-time, large range, large amount data observation of the seafloor. Seafloor observatory network adopts the single-stage medium-voltage DC parallel transmission mode and Dense Wavelength Division Multiplexing (DWDM) technology (Figure 1) [4-5]. It has important practical significance to explore how to separate the submarine cable fault in the parallel mode and distinguish the separate and coupling of different wavelength in space.

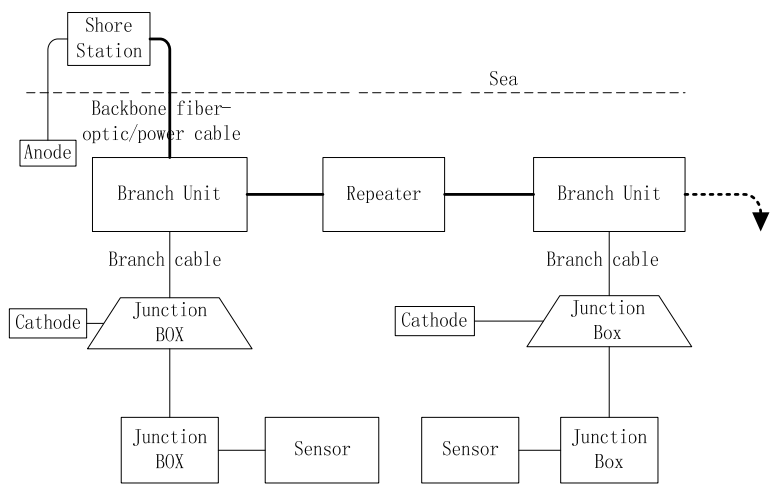

Figure 1. The structure of seafloor observatory network

\section{The STRUCTURE OF BRANCH UNIT}

The seafloor observatory network has two main portions: power supply subsystem and communication subsystem. The power supply portion of the shore station produces medium voltage of $10 \mathrm{kV}$ direct current. The power is transmitted to the main Junction Box for underwater sensor device through fiber-optic/power composite cable, the Branch Unit, the branch cable. The communication portion of the shore station adopts DWDM technology that uses Wavelength Division Multiplex (WDM) to composite different wavelengths into the same fiber to achieve communication with the subsea sensors. The main portion of the repeater is Erbium Doped Fiber Amplifier (EDFA) [6], which is used to enlarge the transmission optical fiber to lengthen the transmission distance.

This paper presents the design of the Branch Units that meet the requirements of power supply and communication part in seafloor observatory system. It realizes the submarine observational network power supply system of parallel connection, and also can isolate the fault of the submarine cable to eliminate the impact of the failure on other parts. Different wavelength signals in the DWDM technology can multiplex and demultiplex under passive condition in the Branch Unit. Branch Unit mainly includes power supply portion and communication portion (Figure 2). The main functions of power supply portion: closes relay switch during the seafloor observatory network starting to realize power supply for the whole system; disconnects switch to isolate the submarine cable fault when it occurs. The main function of communication portion: uses optical add-drop multiplexer (OADM) that could select the signals of local needs and insert the local part of the wave to the trunk line, so as to realize local and main line of communication. 


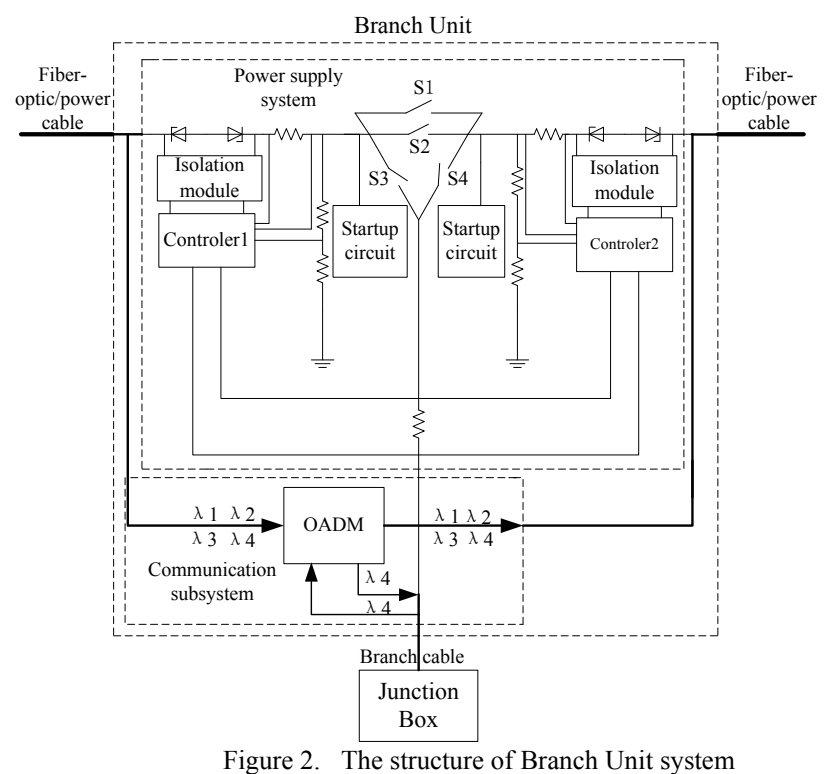

III. HaRdware Design Of Branch Unit

\section{A. Design of communication portion}

In order to improve the reliability of the Branch Unit, the Branch Unit does not have a power transformer. Thus, the communication section of the Branch Unit should select the passive OADM. OADM is one of the key components of the DWDM optical network. This is a type of optical node, which is generally used for the construction of optical telecommunications networks. OADM mainly includes the fiber grating and the optical circulator that achieve the required optical add/drop function. "Add" and "drop" here refer to the capability of the device to add one or more new wavelength channels to an existing multi-wavelength WDM signal, and/or to drop (remove) one or more channels, passing those signals to another network path.

\section{B. Design of power supply portion}

The design lifetime of seafloor observatory network is generally more than 25 years, so Branch Unit must be extremely reliably. In order to improve the Branch Unit reliability, and simplify the Branch Unit design, shore station outputs different voltages to communicate with Branch Unit. Power supply system of seafloor observatory network has five basic operating modes: startup mode, normal operation mode, failure mode, fault location mode, and fault isolation mode [7].The Branch Unit switching circuit is autonomous and mainly works in startup mode, normal mode and fault isolation mode. According to different voltage values, Branch Unit enters different operation modes.

Branch Unit system mainly includes control circuit, startup circuit, zener diode, latch-type vacuum switch, and so on. This paper selects ultra-low-power CC2530 single-chip microcomputer as the processor. The CC2530 chip integrates 8-channel 14-bit A/D to imitate digital conversion, which could accept single-ended and differential input. So, it is easy to collect measurement signal of current and voltage.

\section{The design of startup circuit}

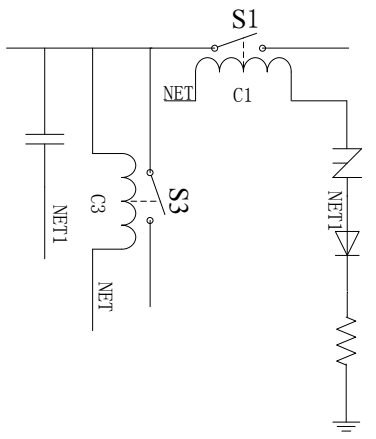

Figure 3. Startup circuit

The function of the startup mode is to close all vacuum switches to start the entire seafloor observatory network in normal operation mode. The output voltage of shore station is lower than $+5 \mathrm{kV}$ in startup mode which is negative $10 \mathrm{kV}$ in normal operation mode. The main components of the startup circuit are shown in Figure 3. The main components are latch-type vacuum switch, Silicon Diode for Alternating Current (SIDAC), a resistance, bypass capacitor, etc. Assume that the shore station is on the left side of the startup circuit. Because the voltage is positive, the bypass capacitor at the left side vacuum switches charges. When the voltage across the bypass capacitor exceeds the trigger voltage of the SIDAC, the bypass capacitor discharges its energy. The current flows through closed solenoid $\mathrm{C} 1$ and $\mathrm{C} 3$, so the vacuum switches $\mathrm{S} 1$ and $\mathrm{S} 3$ are closed. After S1 closes, the S2 and S4 also close. The current reaches next Branch Unit; the startup circuit repeats the same startup procedures until all the Branch Units are closed. The total startup time of a Branch Unit is $500 \mathrm{~ms}$. Startup time is determined by the size of the capacitor and the current limiting resistor. After all the vacuum switches closed, seafloor observatory network is connected, and the system begins to work properly. In addition, the shore station voltage reaches to negative $10 \mathrm{kV}$.

\section{The design of CC2530 power}

The construction cost of seafloor observatory network is high. It is also difficult and expensive to maintain seafloor observatory network. In order to reduce the design cost of Branch Unit, the Branch Unit does not have a power transformer. The zener diodes supply power for controller CC2530. A zener diode is a diode which allows current to flow in the forward direction in the same manner as an ideal diode, but will also permit it to flow in the reverse direction when the voltage is above a certain value known as the breakdown voltage. When the voltage reaches the breakdown voltage, a reverse-biased zener diode will exhibit a controlled breakdown and allow the current to keep the voltage across the zener diode close to the zener breakdown voltage. Therefore, the zener diode is used as a voltage reference element, or a voltage stabilizer. Zener diodes can be used to output a stabilized voltage with low ripple under varying load current conditions. So zener diode can provide power for the controller. A zener diode with a zener breakdown voltage of $5.1 \mathrm{~V}$ connects the transmission network in the reverse-biased direction, and will exhibit a 
voltage drop of very nearly $5.1 \mathrm{~V}$ across a wide range of reverse currents. The control circuit could obtain stable $5 \mathrm{~V}$ voltage though the $5 \mathrm{~V} / 5 \mathrm{~V}$ voltage isolation module. The chip IMP1117-3.3 transforms $5 \mathrm{~V}$ to $3.3 \mathrm{~V}$ for controller power supply. Power section circuit is shown in Figure 4.

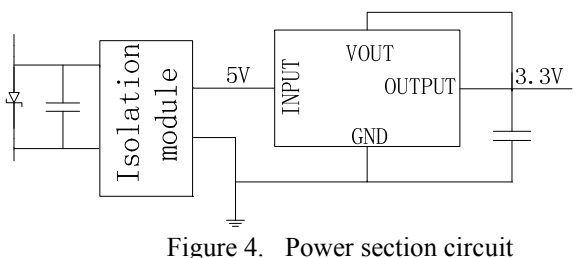

\section{E. The design of voltage and current measurement}

The zener diode provides power for Branch Unit, but its power is too limited to drive current sensors and voltage sensors. Therefore, resistance divider voltage method and sampling resistance method are used to measure cable voltage and able current respectively. Because the seafloor observatory network operating voltage is negative $10 \mathrm{kV}$, this paper chooses Precise Cermet Trimmer Potentiometers to divide voltage. Otherwise, auxiliary voltage-sharing capacitors are used to make the voltage distribution uniform. The sampling resistance measurement is simple in structure, is easy to install and also is not affected by external interference. Since the resistor is connected in series to the circuit, in order to monish power loss, this design should select the smallest resistance in the case of ensuring measurement accuracy. This design reduces the cost of the Branch Unit, simplifies the circuit design, and improves the stability of the Branch Unit.

\section{F. The design of relay switching circuit}

Branch Unit adopts twin coil latching relay to control the backbone power supply circuit and branch power supply circuit. Panasonic DE1a-L2-5V relay switch was used in the experiment. Its relay switch has two operating solenoids; one for closing and one for opening. Self-locking function of relay switch further reduces the power consumption of Branch Unit. In the process of seafloor observatory network startup, the relay switch is closed when the current flows in closing solenoid. In the fault isolation mode, the controller in each Branch Unit uses an isolation algorithm to determine the fault location. The CC2530 chip has two high drives general I/O port P1.0 and P1.1 whose drive capability can reach $20 \mathrm{~mA}$. The two I/O port connect with chip ULN2803 that could stable drive relay solenoid. ULN2803 has the eight NPN Darlington connected transistors in this family of arrays. The ULN2803 is designed to be compatible with standard TTL families. Collector output current can reach $500 \mathrm{~mA}$. One end of the load of the relay solenoid is normally connected to the power supply, and the other end is normally connected to a ULN2803 output terminal.

\section{G. The software design of Branch Unit}

Seafloor observatory network has a perfect Power Monitoring and Control System (PMACS) to ensure that the system can operate stably. PMACS integrates a number of system analysis tools, such as State Estimation, Topology Identification, and Fault Location, and monitors the seafloor observatory network in a real-time environment [8]. When fault occurs, the PMACS goes into the fault locating mode. Then PMACS estimates the voltage that shore station need to output based on the different fault position.

The cable fault is in the branch cable and backbone cable. In the fault isolation mode, shore station outputs voltage less than the starting voltage $-5.2 \mathrm{kV}$ of the DC-DC transformer. This moment, the current only exists in cable fault place. So, the controllers of Branch Unit can judge which branch cable has fault based on current flow. And then the control disconnects relay switch to isolate the fault.

Backbone cable fault model is shown in Figure 5. The short circuit fault appears between Branch Units B and C. The shore station is at the left of Branch Unit A. The current flows from Branch Unit $A$ to $B$, so $V_{A}>V_{B}$. Branch Unit delay time is determined by the measuring voltage and the assumed time constant, as $\mathrm{T}=\mathrm{V} * \lambda$. Thus, the Branch Unit $B$ closest to the fault will open switch first. After the faulty cable is isolated from the network, the Branch Unit A will be out of the fault isolation mode. If Branch Unit B fails to open switch, the current will flow the Branch Unit A. Branch Unit A will react in the same way. Program flow of Branch Unit fault isolation model is shown in Figure 6.

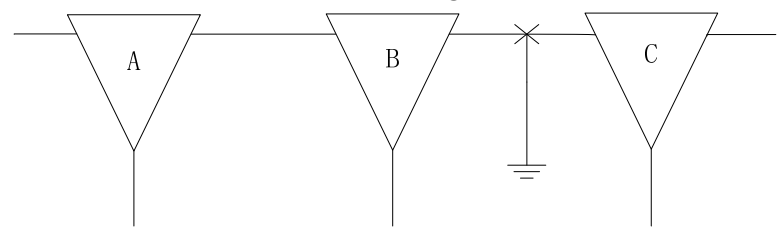

Figure 5. Backbone cable model

\section{BRANCH UNIT SySTEM TEST}

The low voltage Branch Unit was designed based on the above-mentioned principle (Figure 7). Instead of the actual high voltage power supply, DC 600 voltage power supply is used in lab environment. RS232 serial interface is used to show working mode of the Branch Unit.

The main objectives of the lab tests were: to test that the zener diode could provide power for Branch Unit circuit; to test that the capacitor is charged to the break over voltage of the SIDAC and the vacuum switches are closed; to test that the Branch Unit could change automatically work modes according to the different voltages (Figure 8); to test that Branch Unit could disconnect vacuum switch in the fault isolation mode.

\section{CONCLUSION}

This paper describes the general structure and important components of the Branch Unit that could be used in seafloor observatory network. The Branch Unit has two portions: the communication portion and the power supply portion. The Branch Unit that can be used in low voltage is designed. The experiment realizes the basic function of the Branch Unit and verifies its practicality and reliability. These works make it easy to design Branch Unit under high voltage. 


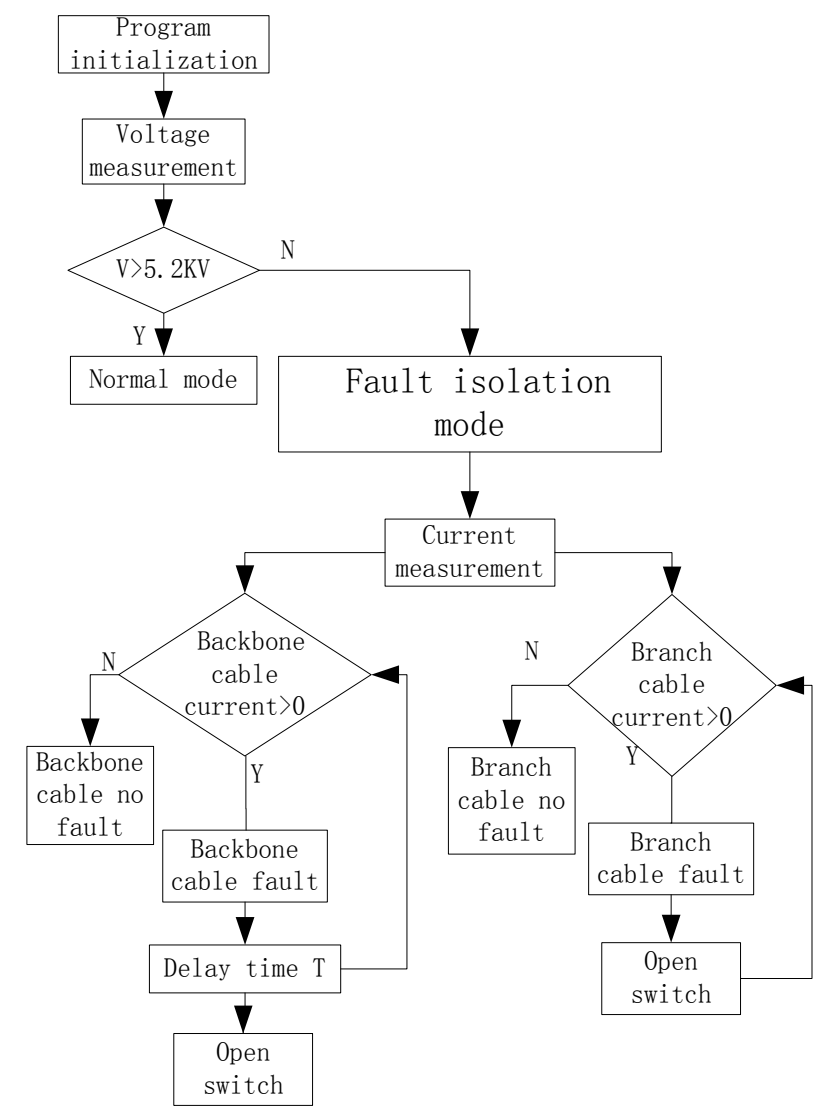

Figure 6. Branch Unit fault isolation model program flow diagram

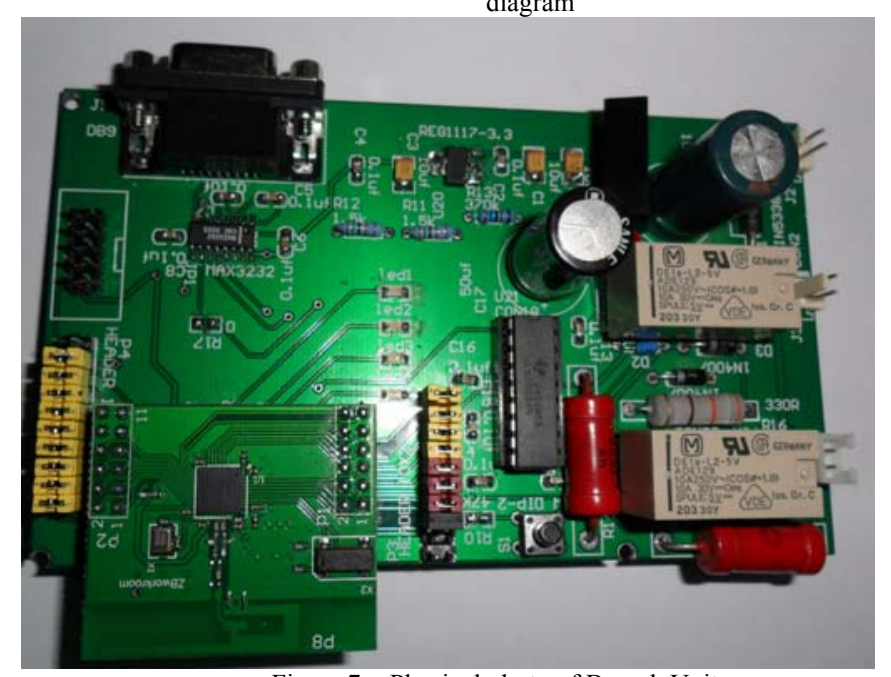

Figure 7. Physical photo of Branch Unit
Start 034V 0.16A1 $0.16 \mathrm{~A} 2$ Start 034V $0.16 \mathrm{AA} 10.16 \mathrm{A2}$ Start 034V 0.16A1 $0.16 \mathrm{AZ}$ Start 034V 0.16A1 0.16A2 Start 034V 0.16A1 0.16A2 Start 034V 0.16A1 0.16A2

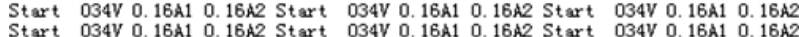

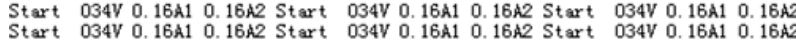

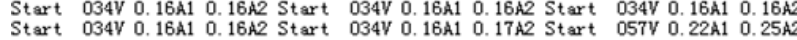

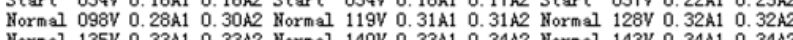
Norm al 135V Normal 150V $0.35 \mathrm{A1} 0.35 \mathrm{A2}$ Normal 151V $0.35 \mathrm{~A} 10.35 \mathrm{A2}$ Normal $151 \mathrm{~V} 0.35 \mathrm{~A} 10.35 \mathrm{A2}$ Normal $151 \mathrm{~V} \quad 0.35 \mathrm{A1} \quad 0.35 \mathrm{A2}$

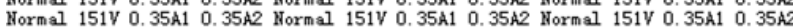

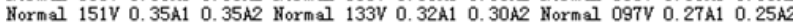
Normal 081V $0.24 \mathrm{~A} 10.24 \mathrm{~A} 2 \mathrm{Fault} 072 \mathrm{~V} 0.23 \mathrm{~A} 10.23 \mathrm{~A} 2 \mathrm{Fault} 073 \mathrm{~V}$ 0.23A1 $0.23 \mathrm{Az}$

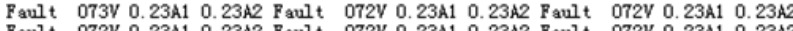
$\begin{array}{llllllllllll}\text { Fault } & 072 \mathrm{~V} & 0.23 \mathrm{~A} 1 & 0.23 \mathrm{~A} 2 & \mathrm{Fault} & 072 \mathrm{~V} & 0.23 \mathrm{~A} 1 & 0.23 \mathrm{~A} 2 & \mathrm{Fault} & 072 \mathrm{~V} & 0.23 \mathrm{~A} 1 & 0.23 \mathrm{AL}\end{array}$ Pault OTV $0.23 A 10.23 A 2$ Fault $12 V 0.23 A 10.23 M 2$ Pault OTV $0.23 A 10.23 A 2$

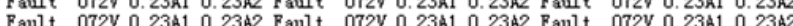

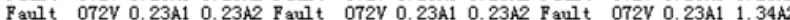
Fault 072V 0.23A1 1.34A2 Fault 072V $0.23 \mathrm{~A} 11.34 \mathrm{~A} 2 \mathrm{Fault} 072 \mathrm{~V} 0.23 \mathrm{~A} 11.34 \mathrm{AL}$ Fault 072V 0.23A1 1.34A2 Fault 072V 0.23A1 $1.34 \mathrm{~A} 2 \mathrm{Fault}$ 072V $0.23 \mathrm{~A} 11.34 \mathrm{AL}$

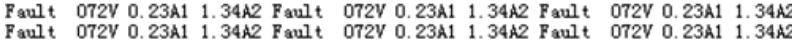

Figure 8. Work modes switch

\section{ACKNOWLEDGMENT}

The project is supported by the National Science and Technology Support Program (the Development of Intelligent Surface Oil Spill Disposal Platform and Complete sets of Equipment: NO.2012BAC14B00).

\section{REFERENCES}

[1] L. Shuai, M.A. El-Sharkawi,H. kirkham, and B.W. Howe, "NEPTUNE Power System: Startup Power Supply for $10 \mathrm{kV}$ to $400 \mathrm{~V}$ Dc-Dc Converters," Proc. Applied Power Electronics Conference and Exposition(APEC06), April.2006, pp.1385-1389.

[2] P. Fairl, "Neptune rising[undersea observatory]," Spectrum, vol. 42 (11), pp.38-45,2005.

[3] R. Person,L. Beranzoli, C. Berndt, el al. "The European Deep Sea Observatories Network of Excellence ESONET," Proc. OCEANS. June. 2007, pp.1-6.

[4] B. Howe, H. Kirkham, V. Vorperian and P. Bower, "The Design of the NEPTUNE Power System," Proc. OCEANS, 2001. MTS/IEEE Conference and Exhibition, August 2002, pp.1373-1380.

[5] S.T. Lentz, "The NEPTUNE Canada Communications Network," Proc.OCEANS.Feb.2008, pp.1-5.

[6] C. Konishi, T. Yoshida, S. Hamada, K. Asahi, and S. Fujita, "Dynamic gain-controlled erbium-doped fiber amplifier repeater for WDM network," Proc. Optical Fiber Communication (OFC 97), Feb.1997, pp.18-19.

[7] Lin Chen-Ching, K. Schneider, H. Kirkham, B. Howe, "State Estimation for the NEPTUNE Power System," Proc. Transmission and Distribution Conference and Exposition, Sept.2003, pp.748-754.

[8] Chan Ting, Liu Chen-Ching,B.M.Howe,H.Kirkham. "Fault Location for the NEPTUNE Power System," Power System, vol.22(2), pp. $522-531,2007$ 